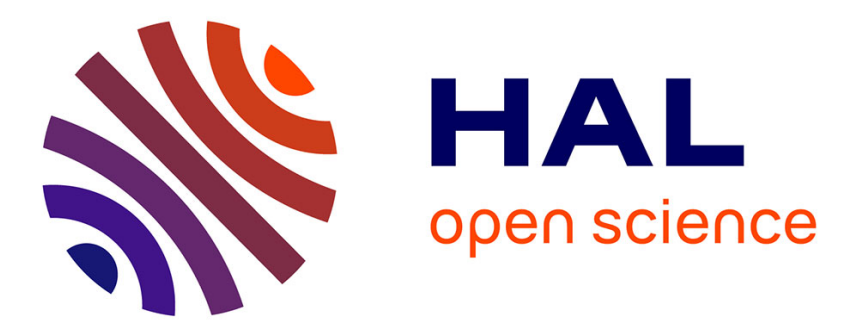

\title{
Transparency-optimal passivity layer design for time-domain control of multi-DoF haptic-enabled teleoperation
}

\author{
Olmo A Moreno Franco, Joao Bimbo, Claudio Pacchierotti, Domenico \\ Prattichizzo, Davide Barcelli, Gianni Bianchini
}

\section{To cite this version:}

Olmo A Moreno Franco, Joao Bimbo, Claudio Pacchierotti, Domenico Prattichizzo, Davide Barcelli, et al.. Transparency-optimal passivity layer design for time-domain control of multi-DoF haptic-enabled teleoperation. IROS 2018 - IEEE/RSJ International Conference on Intelligent Robots and Systems, Oct 2018, Madrid, Spain. pp.1-7. hal-01851708

\section{HAL Id: hal-01851708 \\ https://hal.inria.fr/hal-01851708}

Submitted on 30 Jul 2018

HAL is a multi-disciplinary open access archive for the deposit and dissemination of scientific research documents, whether they are published or not. The documents may come from teaching and research institutions in France or abroad, or from public or private research centers.
L'archive ouverte pluridisciplinaire HAL, est destinée au dépôt et à la diffusion de documents scientifiques de niveau recherche, publiés ou non, émanant des établissements d'enseignement et de recherche français ou étrangers, des laboratoires publics ou privés. 


\title{
Transparency-optimal passivity layer design for time-domain control of multi-DoF haptic-enabled teleoperation
}

\author{
Olmo A. Moreno Franco ${ }^{1}$, Joao Bimbo ${ }^{1}$, Claudio Pacchierotti ${ }^{2}$, \\ Domenico Prattichizzo ${ }^{1,3}$, Davide Barcelli ${ }^{3}$, and Gianni Bianchini ${ }^{3}$
}

\begin{abstract}
This paper presents a novel optimization-based passivity control algorithm for haptic-enabled bilateral teleoperation systems involving multiple degrees of freedom. In particular, in the context of energy-bounding control, the contribution focuses on the implementation of a passivity layer for an existing time-domain scheme, ensuring optimal transparency of the interaction along subsets of the environment space which are preponderant for the given task, while preserving the energy bounds required for passivity. The involved optimization problem is convex and amenable to real-time implementation. The effectiveness of the proposed design is validated via an experiment performed on a virtual teleoperated environment.
\end{abstract}

\section{INTRODUCTION}

In haptic-enabled teleoperation, the primary concern is to provide a stable and transparent operation of the system. It is well known that haptic feedback can lead to unstable and therefore unsafe behavior of the overall system due to factors such as communication latency in the loop, hard contacts, and relaxed user grasps. Such behavior must be avoided, especially in fields where safety is a paramount and non-negotiable requirement (e.g., medical robotics) [1]. Transparency can be defined as the match between the impedance of the teleoperated environment and the one perceived by the operator [2]. This property allows for achieving a good illusion of telepresence [3], [4]. Indeed, haptic stimuli play an important role in improving the performance of teleoperation systems in terms of task completion time [5], [6], accuracy [7], [8], peak and mean force [9]. Therefore, guaranteeing stability while preserving transparency has always been a prominent challenge of the fields of robotics and haptics. To this purpose, a great variety of control design approaches have been proposed. In this context, passivity theory [10], [11] has been recognized as an effective tool for achieving stable interaction. In [12], [13], passivity is analyzed in the time domain in terms of energy levels of system components. In [14], the problem of making a delayed communication channel passive is addressed. Energy-bounding algorithms to guarantee passivity of the teleoperation loop have been proposed in [15], [16], [17]. Along the same line, in [18], a two-layer control scheme is proposed, in which a transparency layer computes the ideal

\footnotetext{
${ }^{1}$ Olmo A. Moreno F., Joao Bimbo and Domenico Prattichizzo are with Advanced Robotics Department, Istituto Italiano di Tecnologia, 16163, Genova, Italy \{olmo.moreno, joao.bimbo\}eit. it

${ }^{2}$ Claudio Pacchierotti is with CNRS, Univ Rennes, Inria, IRISA, 35042 Rennes Cedex, France claudio.pacchierotti@irisa.fr

${ }^{3}$ Gianni Bianchini, Davide Barcelli, and Domenico Prattichizzo are with the Dipartimento di Ingegneria dell'Informazione e Scienze Matematiche, Università degli Studi di Siena, 53100 Siena, Italy \{prattichizzo, barcelli,giannibi\}@diism.unisi.it
}

forces to be actuated at both the master and slave sides, while a passivity layer modulates such forces when necessary to avoid violations of the passivity condition, thus guaranteeing stability at the price of a temporary loss of transparency. More recently, in [19] the authors introduced a passivity-based interactive control architecture based on the port-Hamiltonian framework. Most of the cited time-domain approaches employ the concept of energy tanks to enable the use of the (virtual) energy circulating in the controlled system in a flexible and passivity-preserving way.

This work builds upon the two-layer architecture proposed in [18]. In that paper, the design of the passivity layer does not explicitly account for the amount of transparency that is lost due to the stabilizing effect. This issue is of fundamental importance especially in complex teleoperation tasks that involve multiple degrees of freedom (DoF). Indeed, for a particular configuration of a given task, it may be important to conserve transparency in terms of fidelity of the rendered force along some subset of the task space, while other components may be significantly altered in order to preserve passivity without compromising the overall task performance. The aim of this paper is to present an optimization-based design of the passivity layer which guarantees the maximum possible degree of transparency along subsets of the environment space that are preponderant for the given task at a given time, while preserving the energy bounds that are required to guarantee passivity. The optimal rendered force is computed via the solution of a convex quadratic program which is characterized by modest computational complexity and amenable to implementation in real time.

The paper is organized as follows. In Section II some preliminary concepts are recalled. The control architecture this work is based on is summarized in Section III. The proposed optimal control design is presented in Section IV. Experimental validation of the approach is performed in Section V. Finally, conclusions are drawn in Section VI.

\section{A. Notation}

We denote the continuous time index as $t$ and the discrete time index as $k$. For a continuous-time signal $q(t)$, we denote its sampling $q\left(k T_{s}\right)$ with $q(k)$ without ambiguity, being $T_{s}$ the sampling period. The interval from time $k-1$ to time $k$ is indicated as $\bar{k} . H(\bar{k})$ denotes a signal $H$ being held constant during $\bar{k}$. For a vector or matrix $v, v^{\prime}$ indicates its transpose. The notation $v \odot w$ is used to denote the component-bycomponent product of $v$ and $w .\|v\|$ is the Euclidean norm of $v$. 


\section{Preliminaries}

\section{A. Transparency}

Transparency is defined as the full display of the environment impedance to the human operator when he/she interacts with the robot [20], [21]. As defined in [22], ideal transparency is achieved when the generalized forces $\tau_{s}$ and velocities $\dot{q}_{s}$ at the slave side are equally reflected in the forces $\tau_{m}$ and velocities $\dot{q}_{m}$ at the master side, modulo a suitable scaling and an intrinsic time delay.

\section{B. Passivity}

Stability of the teleoperation chain is a key requirement in order to ensure a safe interaction at both the master and the slave sides. This property may be compromised by several factors such as relaxed user grasp, stiff contacts in the environment, and communication delays.

Passivity represents a viable solution to the stability preservation problem. Indeed, the interaction between passive systems is guaranteed to be stable, and properly combining passive systems results in a passive system [10]. Moreover,the human operator has been shown to preserve stability when interacting with a passive system [23]. Hence, ensuring passivity of all components of the telemanipulation system is a convenient sufficient condition for stability of the interaction at all levels.

For a generic component $R$ of a mechanical teleoperation system, let $H_{R}(t)$ denote its total internal energy. Passivity of $R$ boils down to the condition that $H_{R}(t)$ never exceeds its initial value $H_{R}(0)$. Assuming without loss of generality a zero-energy initial condition, $R$ is passive if

$$
H_{R}(t)=\int_{0}^{t} \tau_{R}^{\prime}(\sigma) \dot{q_{R}}(\sigma) d \sigma \geq 0 \quad \forall t \geq 0,
$$

where $\tau_{R}(t)$ and $\dot{q_{R}}(t)$ represent the applied external forces and generalized velocities, respectively.

Following [18], the total energy $H_{T}(t)$ of the system can be decomposed as

$$
H_{T}(t)=H_{M}(t)+H_{S}(t)+H_{C}(t),
$$

where $H_{M}(t), H_{S}(t)$ and $H_{C}(t)$ denote the energy levels pertaining to the master side, the slave side, and the communication channel, respectively. From (1), passivity of the overall system is therefore achieved if the controller is able to regulate the system in order to preserve the condition

$$
H_{T}(t) \geq 0 \quad \forall t \geq 0 .
$$

\section{CONTROL ARChitecture}

The present work builds upon the energy monitoring control architecture presented in [18]. With reference to Fig. 1, the overall system is made of several layers.

The physical layer represents the user/haptic device and robot/environment interactions. The generalized forces and displacements at the master [slave] side are denoted by $\tau_{m}(k)\left[\tau_{s}(k)\right]$ and $q_{m}(k)\left[q_{s}(k)\right]$, respectively.

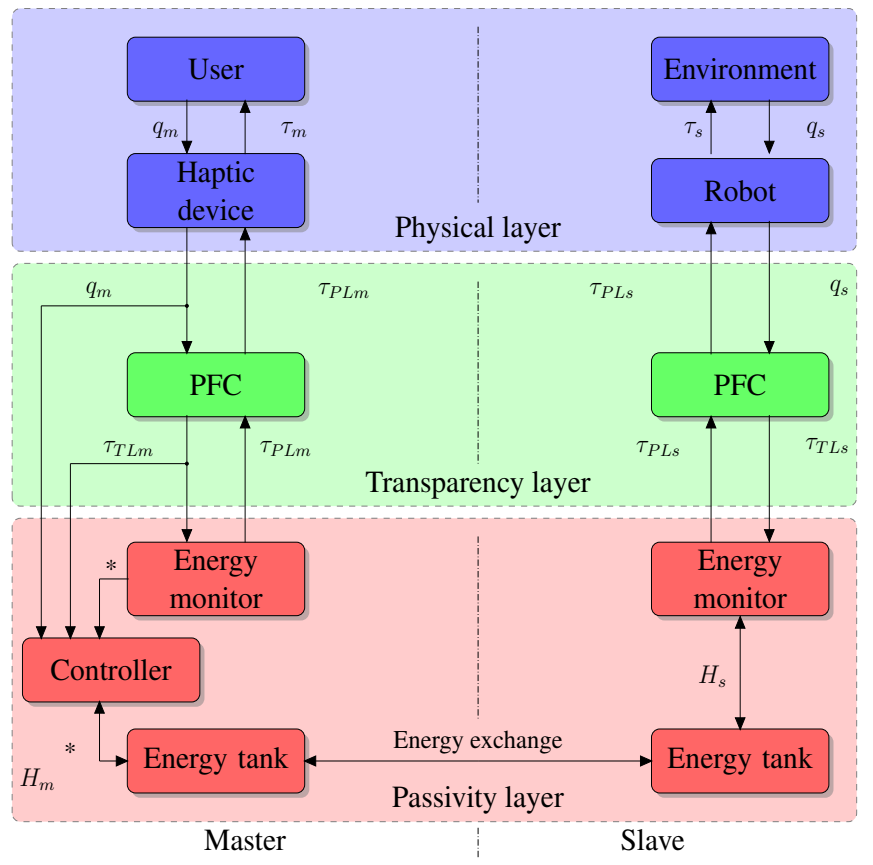

Fig. 1. Controller architecture block diagram. The architecture of the controller is divided in three areas: A) the physical layer that defines forces as inputs and position as outputs for the devices. B) the transparency layer that computes position to force control. C) the passivity layer which computes the damping correction (only on the master side) and energy flow control.

The transparency layer implements a position force controller (PFC) and interacts between the physical and passivity layer exchanging information on forces and positions.

A standard implementation of the PFC is as follows:

$$
\begin{aligned}
\tau_{T L m}(k) & =\tau_{e}(k-T) \\
\tau_{T L s}(k) & =-K_{p}\left(q_{m}(k-T)-q_{s}(k)\right)-K_{d} \dot{q}_{s}(k)
\end{aligned}
$$

where $\tau_{e}(k)$ si the measured interaction force at the master side, while $K_{p}$ and $K_{d}$ are suitable proportional and derivative controller gains, and $T$ is the master-slave communication delay (possibly time-varying). The force vectors $\tau_{T L m}(k)$ and $\tau_{T L s}(k)$, once actuated at the respective sides, ensure full transparency to the teleoperation system. Such transparency might not be always achieved in a passive manner. The role of the passivity layer is to suitably modulate the forces $\tau_{T L}(k)$ generated by the PFCs at both sides in order to preserve passivity of the overall system, i.e., to ensure that (2) holds at all times. Due to the impossibility of monitoring the overall energy $H_{T}$ in real time due to the presence of delays, in [18] the following paradigm is adopted. A virtual energy tank $H$ characterized by an energy level $H(k)$ is introduced at each side, Each tank can exchange virtual energy with its counterpart at the other side and functions as the energy budget available for performing the appropriate (master or slave) force control action. When the tank level at one side is detected as being low, a correction to the actuated force $\tau_{T L}(k)$ is applied in order for the tank level not to drop below zero, therefore preserving passivity.

The passivity layer also integrates an energy exchange protocol whose role is to transfer virtual energy packets 
between the master and slave tanks according to a suitable algorithm. The purpose of this protocol is to balance energy levels at both sides, so as to relax the conservative condition that both tank levels be positive in order to guarantee overall passivity. The details on the latter component are out of the scope of this work and the standard implementation of [18] is used here. In this section, we describe the passivity layer without specific reference to the master or the slave side, as it can be implemented in either, and stick with the notation used in [18].

Let $q(k)$ be the sampled generalized device displacement, and denote with $\tau_{r}(\bar{k})$ the actuated force during $\bar{k}$, which is held constant since a zero-order-hold is used. The energy loss in tank $H$ during $\bar{k}$ is therefore given by

$$
\Delta H(k)=\tau_{r}^{\prime}(\bar{k})(q(k)-q(k-1)),
$$

Hence, the tank level after the control action has been performed amounts to

$$
H(k)=H(k-1)-\Delta H(k) .
$$

Taking into account a possible virtual energy exchange amounting to $H_{ \pm}(k)$, performed according to the exchange protocol, the energy tank level at the end of the time interval $\bar{k}$ becomes

$$
H(\overline{k+1})=H(k)+H_{ \pm}(k) .
$$

The tank level $H(\overline{k+1})$ in (7) represents the available amount of energy to perform the force actuation task during the time interval $\overline{k+1}$. In [18], the passivity layer is implemented as a curtailed version $\tau_{P L}(k)$ of the force feedback $\tau_{T L}(k)$, computed according to the value of $H(\overline{k+1})$. In particular, the following two strategies are considered.

1) Simply cut-off all forces if there's no energy left, i.e.,

$$
\tau_{P L}(k)= \begin{cases}0 & \text { if } H(\overline{k+1}) \leq 0 \\ \tau_{T L}(k) & \text { otherwise }\end{cases}
$$

2) Provided the teleoperation system has only a single DoF, an estimate of the energy required for the control action in $\overline{k+1}$ is given by

$$
\hat{H}(\overline{k+1})=\tau_{r}(\overline{k+1}) \dot{q}(k) T_{s},
$$

where $\tau(\overline{k+1})$ is the prospective actuated force. Based on this and on the available energy, a limitation on $\tau(\overline{k+1})$ is as

$$
\tau_{\max }(k)=\frac{H(\overline{k+1})}{|\dot{q}(k)| T_{s}} .
$$

The curtailed force $\tau_{P L}(k)$ is then computed as $\tau_{P L}(k)= \begin{cases}0 & \text { if } H(\overline{k+1}) \leq 0 \\ \operatorname{sgn}\left(\tau_{T L}(k)\right) \min \left(\left|\tau_{T L}(k)\right|, \tau_{\text {max }}(k)\right) & \text { otherwise. }\end{cases}$

Finally, a certain amount of virtual damping force $\tau_{d}$ is added at the master side to prevent total tank depletion.

In [18], the problem of suitably shaping $\tau_{P L}(k)$ in the multi-DoF case, depending on the task and in order to preserve transparency under the passivity constraint is left as an open question.

\section{OPTIMAL PASSIVITY LAYER DESIGN}

In this paper, we propose an optimization-based design of the passivity layer which addresses the multi-DoF case and minimizes the loss of transparency on subsets of the task space which are relevant to the given task, while preserving passivity.

Similarly to case 2) above, we consider the estimated energy loss as a function of the prospective actuated force $\tau_{r}(\overline{k+1})$ as

$$
\Delta \hat{H}(\overline{k+1})=\tau_{r}^{\prime}(\overline{k+1}) \dot{q}(k) T_{s} .
$$

Therefore, an estimate of the tank level after the control action has been performed is given by

$$
\hat{H}(k+1)=H(\overline{k+1})-\tau_{r}^{\prime}(\overline{k+1}) \dot{q}(\bar{k}) T_{s} .
$$

Let $H_{\min }(k)>0$ be a possibly time-varying threshold level, chosen by the designer, corresponding to the amount of energy to be left in the tank after $\tau_{r}(\overline{k+1})$ has been applied during $\overline{k+1}$. In order for such a level to be guaranteed, according to the estimate in (11), the following constraint must hold:

$$
\hat{H}(k+1)=H(\overline{k+1})-\tau_{r}^{\prime}(\overline{k+1}) \dot{q}(\bar{k}) T_{s} \geq H_{\min }(k) .
$$

It is easily seen from (12) that if $H(\overline{k+1})-\tau_{T L}^{\prime}(k) \dot{q}(k) T_{s} \geq H_{\min }(k)$, then the unmodified $\tau_{T L}(k)$ can be safely actuated (i.e., $\tau_{P L}(k)=\tau_{T L}(k)$ ) and therefore perfect transparency can be achieved. To address the situation in which this is not possible, $\tau_{P L}(k)$ is computed as the solution of an optimization problem. To this purpose, we find it convenient to implement the effect of the passivity layer as a force correction in the form of a suitable amount of virtual damping. In particular, we define $\tau_{P L}(k)$ as

$$
\tau_{P L}(k)=\tau_{T L}(k)+\tau_{T L C}(k)
$$

where $\tau_{T L C}(k)$ has the form

$$
\tau_{T L C}(k)=-B(k) \dot{q}(k)
$$

In (14), $B(k)$ is a symmetric matrix to be computed in an optimal fashion.

To formulate the optimization problem, let $\mathcal{S}_{i}(k), i=$ $1, \ldots m$ be a suitable set of subspaces of the task space, depending on the given task and possibly also on the time index $k$. Let us assign a priority index $p_{i}(k) \geq 0$ to each subspace $\mathcal{S}_{i}(k)$, also depending on the current task configuration. The idea of associating each subspace to a priority index is quite simple: the higher the priority $p_{i}(k)$, the stricter the requirement that the projection on $\mathcal{S}_{i}(k)$ of the optimal rendered force $\tau_{P L}(k)$ be as close as possible to the corresponding projection of $\tau_{T L}(k)$. Let $A_{i}(k)$ be a matrix whose columns form a basis of $\mathcal{S}_{i}(k)$. The projection of the force correction $\tau_{T L C}(k)=\tau_{P L}(k)-\tau_{T L}(k)$ on $\mathcal{S}_{i}(k)$ is given by

$$
\Pi_{\mathcal{S}_{i}}\left(\tau_{T L C}(k)\right)=T_{i}(k) \tau_{T L C}(k),
$$

where the projection matrix $T_{i}(k)$ reads

$$
T_{i}(k)=A_{i}(k)\left[A_{i}^{\prime}(k) A_{i}(k)\right]^{-1} A_{i}^{\prime}(k) .
$$


Based on the arguments above, it is natural to define the following functional to be minimized:

$$
J(B(k))=\sum_{i=1}^{m} p_{i}(k)\left\|T_{i}(k) \tau_{T L C}(k)\right\|^{2}
$$

where $\tau_{T L C}(k)$ is as in (14) and the decision variables are represented by the entries of the damping matrix $B(k)$. The minimization of (16) must be carried out under the constraint (12). Furthermore, it must be ensured that the sign of all components of $\tau_{P L}(k)$ be the same as the corresponding components of $\tau_{T L}(k)$ as a result of the force correction in (13)-(14) corresponding to the minimum of $J(B(k))$. This constraint prevents the passivity layer from letting the tank gain energy from inverting the direction of rendered forces with respect to $\tau_{P L}(k)$, thus resulting in an excessive loss of transparency. Said in another way, the maximum allowed correction along each direction must act so as to zero out the rendered force. To ensure the latter condition, the following constraint is required:

$$
\tau_{P L}(k) \odot \tau_{T L}(k)=\left(\tau_{T L}(k)-B(k) \dot{q}(k)\right) \odot \tau_{T L}(k) \geq 0
$$

Motivated by the above observations, we propose the following algorithm as the implementation of the passivity layer.

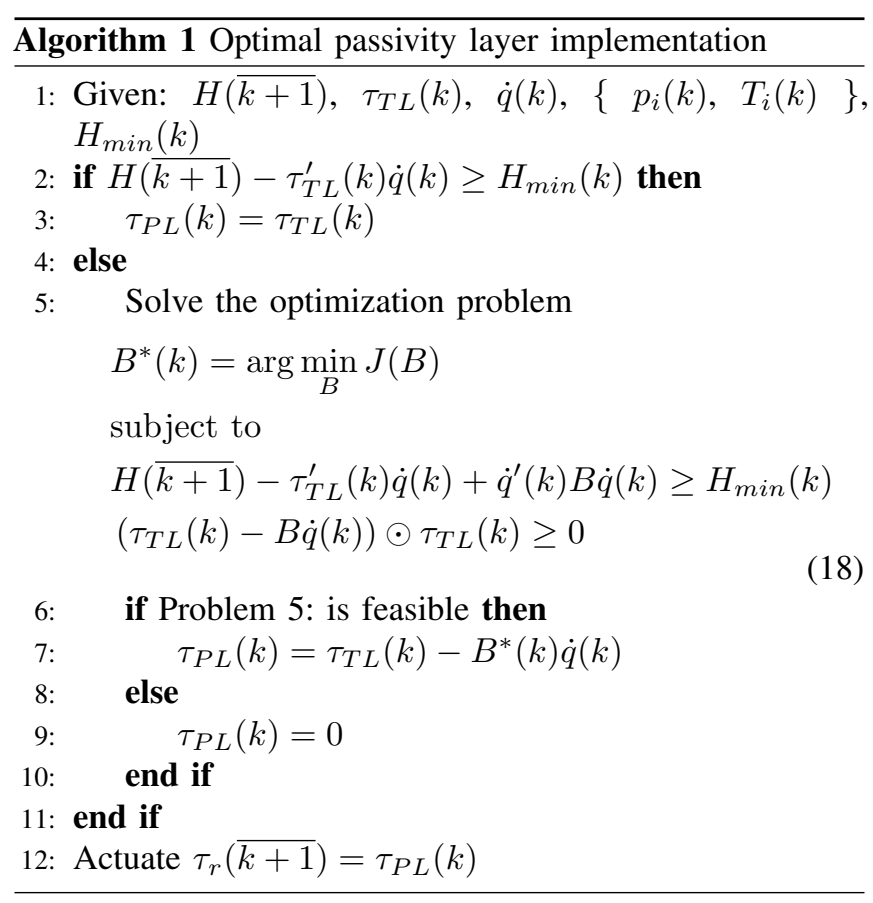

The optimization problem (18) is a convex quadratic program. The global minimum can be efficiently computed using interior point methods. As it will be shown in the experimental section, the method can be implemented without problems on a $3-\mathrm{DoF}$ teleoperation system operating at a sampling frequency of $1 \mathrm{kHz}$.

\section{EXPERIMENTAL EVALUATION}

\section{A. Overview}

To evaluate the feasibility and effectiveness of our Transparency-Optimal Passivity Layer (TOPL) approach, we carried out a palpation experiment. A user with previous experience on haptic devices was asked to palpate a virtual surface and locate an area stiffer than the rest. We evaluated the task performance considering different controller types and experimental conditions, i.e., the delay $T$ in the loop. Fig. 2 shows the experimental setup. A detailed explanation of each experimental component follows.

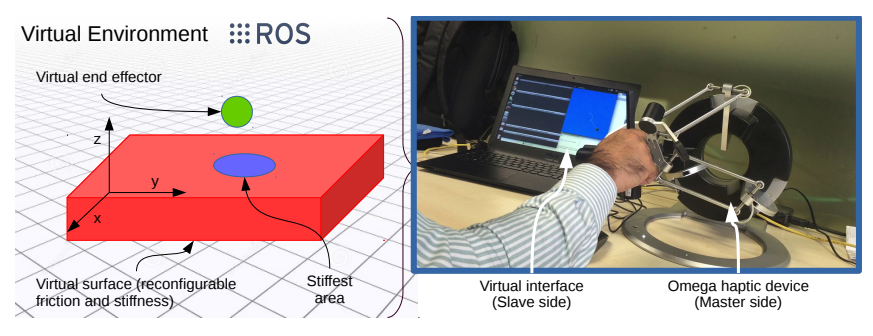

Fig. 2. Experimental set-up. On the left, the virtual environment (slave side) shows a contact area (the red surface) with a stiffness value lower than the virtual "bump" (the blue area). The end effector of the virtual environment is represented by the green sphere, and interacts with the surface, commanded by the master side. On the right side of the picture the Omega haptic device is shown. The virtual environment is an animated interface presented on the screen.

\section{B. Experimental setup}

As shown in Fig. 2, the master side is composed of a human user holding the end-effector of a grounded haptic device, whose position is linked to the one of a virtual end effector in the virtual environment. The user, by controlling the haptic interface, moves the virtual end effector and receives haptic feedback whenever it interacts with the virtual surface.

A stiff circular region of $1 \mathrm{~cm}$ radius is positioned randomly on this virtual surface. Differently from Fig. 2, during the experiment, the user was not provided with any visual information on the position of the stiffer region, and he had to rely only on force cues.

1) Haptic interface: The master side consists of a 6-DoF Omega haptic interface (Force Dimension, $\mathrm{CH}$ ). It has $3 \mathrm{DoF}$ for translations, which are actuated, and $3 \mathrm{DoF}$ for rotations, which are not actuated. To reduce user's fatigue, the Omega haptic device provides gravity compensation.

This impedance haptic interface measures the positions of the end effector grasped by the human operator as an input, and renders forces on the end effector as an output. The haptic control loop operates at $1 \mathrm{kHz}$.

2) Communication Channel: The communication channel collects and distributes the signals between the master and slave sides. Those signals include the actions evaluated by transparency layer on the master side $\tau_{T L m}$ and the slave side $\tau_{T L s}$. They also include the energy exchange information shared between master and slave. Finally, the communication channel introduces a time delay $T$ (see (3)), which affects the forces and energy package distribution between sides. We 
carried out our palpation task simulating three communication delays $T: 1 \mathrm{~ms}, 5 \mathrm{~ms}$, and $10 \mathrm{~ms}$. These delays were added up to the intrinsic delay of the system, which was measured to be, on average, $1.8 \mathrm{~ms}$.

3) Virtual environment: The virtual environment runs on a GNU/Linux machine using the Robot Operative System (ROS) framework. It was rendered using the RVIZ visualization tool, as shown in Fig. 2. It is composed of the virtual surface, which is modeled as a spring with elastic constant $K=1 \mathrm{~N} / \mathrm{cm}$ (red area in Fig. 2). As mentioned before, within this virtual surface, we placed a stiffer 1-cm-radius circular region (blue area in Fig. 2). This stiffer area was modeled as a spring having elastic constant $K_{H}=2 \mathrm{~N} / \mathrm{cm}$. When the end-effector penetrates the virtual surface, the ideal force rendered on the $z$ axis, normal to the surface, is

$$
\tau_{e}(k)=\left\{\begin{array}{cc}
-K_{H}\left(h_{0}(k)-q_{z}(k)\right) & \text { if on the stiffer area } \\
-K\left(h_{0}(k)-q_{z}(k)\right) & \text { otherwise }
\end{array}\right.
$$

where $h_{0}(k)$ is the $z$ position of the uncompressed surface and $q_{z}(k)$ the position of the virtual end effector along the $z$ axis. When moving laterally across the surface, along axes $x$ and $y$, the user is provided with force feedback about the friction of the surface, modeled using a standard Coulomb friction model with coefficient $\mu=0.005$.

\section{Controllers}

1) No Passivity Controller (NPC): The first approach to be tested was a controller that directly transmits the forces and velocities between master and slave, without any concern for passivity.

2) Simple Tank Level Controller (STLC): This controller follows the two-layer energy-based approach, presented by Franken et al. [18], where the force is limited by the energy available in the tank according to (8), and introduces a damping force on the master side. According to [18], the master side damping is implemented as

$$
\begin{aligned}
\tau_{d}(k) & =-d(k) \dot{q}_{m}(k) \\
d(k) & =\left\{\begin{array}{cc}
\alpha\left(H_{d}-H(\overline{k+1})\right) & \text { if } H(\overline{k+1})<H_{d} \\
0 & \text { otherwise }
\end{array}\right.
\end{aligned}
$$

where the damping gain $\alpha$ is set to $100, H_{d}$ is set to 0.2 , and the energy exchange factor $\beta$ described on section V-B.2 of [18] is set to 0.01 .

3) Transparency-Optimal Passivity Layer (TOPL) : The approach described in IV was implemented using the architecture in Fig. 1. In this controller, a dynamic threshold level $H_{\min }(k)$ is implemented as follows:

$$
H_{\text {min }}(k)=H(\overline{k+1})+\eta\left(H_{0}-H(\overline{k+1})\right)\|\dot{q}(k)\|^{2},
$$

where $H_{0}$ represents a reference tank level to be maintained during transparent operation, and $\eta>0$ is a tunable parameter which acts as a proportional controller gain. Equation (21) allows the system to recover energy when the level of energy is below the reference level $H_{0}$, as well as to limit the spending of energy when above. The norm of the current velocity also is weighted in the proportional controller gain in order to limit the damping forces generated by the passivity layer.

Given the aim of the experiment with respect to the virtual teleoperated environment, the subspaces $\mathcal{S}_{i}, i=1,2,3$ have been defined as the three Cartesian axes $x, y, z$. The priorities $p_{i}(k)$ are taken to be constant. The highest priority was assigned to the projection on the $z$ axis, since the perception of the stiffness perpendicular to the surface is fundamental for the scope of the experiment, while friction forces on the horizontal $(x, y)$ plane are assigned lower priorities. The priority values were set as $p_{1}(k)=p_{2}(k)=0.1, p_{3}(k)=0.5$.

\section{Validation}

Before running the palpation experiment, we carried out a simple validation of our setup. A human user was asked to move the virtual end effector across the surface, passing over the stiff circular area. Fig. 3 shows a representative trial, capturing the moment the user passes on the stiffer area of the environment. The forces computed by the transparency layer $\tau_{T L}(k)$ and the ones computed by the passivity layer $\tau_{P L}(k)$ along the $z$ axis are shown in the upper part of Figs. 3a, 3b, $3 \mathrm{c}$. In the lower part of the same figures, we can see the same forces $\tau_{T L}(k)$ and $\tau_{P L}(k)$ for axes $x$ and $y$. In this case, a delay of $1 \mathrm{~ms}$ was present between master and slave. When enforcing no passivity controller, the force measured in the virtual environment is directly provided to the user through the haptic interface, i.e., $\tau_{P L}=\tau_{T L}$ regardless of passivity constraints (see Sec. V-C.1). When enforcing the STLC controller, a damping action is introduced along all directions when the energy tank level is below a predefined threshold. In the proposed TOPL approach, we give a higher priority/importance to vertical forces, which are the most informative for our palpation task, at the expense of losing transparency when rendering the horizontal (friction) forces. In this respect, we can see that in Fig. 3b (STLC) corrective actions are taken along all directions. On the other hand, Fig. $3 \mathrm{c}$ shows that the proposed approach takes significant corrective actions along the non-preferred directions ( $x$ and $y$ ), while it preserves transparency along the preferred direction $z$, which is the most useful for our palpation task.

\section{E. Palpation experiment}

In furtherance of testing the proposed approach, a human subject carried out 48 repetitions of our palpation task. The user was asked to find the location of a $3.14 \mathrm{~cm}^{2}$ stiffer region placed on the virtual surface, as described in Sec. V-B. We compared the performance of the three controllers described in Sec. V-C, each tested when simulating three different communication delays between master and slave $(1 \mathrm{~ms}$, $5 \mathrm{~ms}$, or $10 \mathrm{~ms}$ ). To evaluate the effectiveness of our system in correctly rendering the stiffness of the environment, we registered the accuracy error in detecting the stiffer area within our virtual surface. Fig. 4 shows this result in the nine different experimental conditions. To compare this metric among the conditions, we ran a two-way repeated-measures ANOVA test on the data. Time delay in the communication (1 ms vs. $5 \mathrm{~ms}$ vs. $10 \mathrm{~ms}$ ) and stability controller (no controller, NPC 

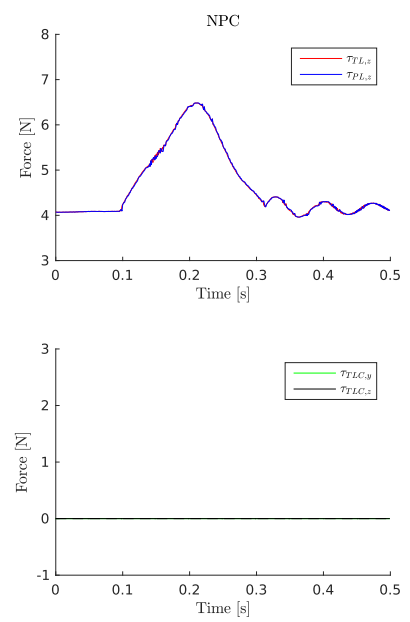

(a) No Passivity Controller (NPC)
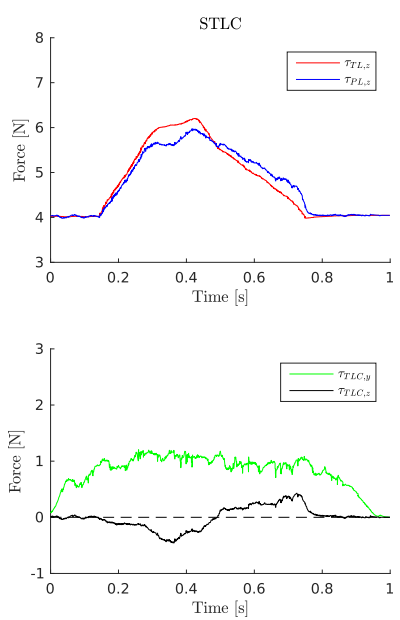

(b) STLC
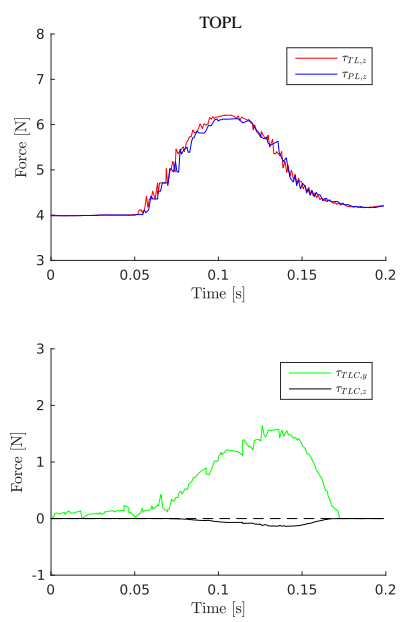

(c) TOPL

Fig. 3. Forces of the three experimental conditions with a pass over the bump. (a) shows the $\tau_{T L, z}$ and $\tau_{P L, z}$ forces when the end effector passes over the bump; as seen, $\tau_{T L, z}$ follows $\tau_{P L, z}$ presenting the most transparent scenario (NPC). (b) presents $\tau_{T L, z}$ and $\tau_{P L, z}$ forces when STLC controller is running, as well the $\tau_{T L C, y}$ and $\tau_{T L C, z}$ forces, that belong to the correction phase. (c) displays the $\tau_{T L, z}$ and $\tau_{P L, z}$ forces when TOPL controller is working, and the damping correction on $\tau_{T L C, y}$ and $\tau_{T L C, z}$ forces.

vs. Franken et al. [18], STLC vs. our proposed approach, TOPL) were treated as within-subject factors. All data passed the Shapiro-Wilk normality test. Interaction effects between the factors were not statistically significant. Mauchly's Test of Sphericity indicated that the assumption of sphericity had been violated for both variables (delay, $\chi^{2}(2)=23.148, p<$ 0.001 ; controller, $\left.\chi^{2}(2)=15.987, p<0.001\right)$. The twoway repeated-measure ANOVA with a Greenhouse-Geisser correction revealed statistically significant difference between the time delays $\left(F_{1.005,6.029}=6.815, p=0.011, a=0.05\right.$, partial $\left.\eta^{2}=0.533\right)$ and stability controllers $\left(F_{1.021,6.125}=\right.$ 17.701, $p<0.001, a=0.05$, partial $\left.\eta^{2}=0.745\right)$. Post hoc analysis with Bonferroni adjustments revealed a statistically significant difference between having $1 \mathrm{~ms}$ vs. $5 \mathrm{~ms}$ delay $(p=0.019)$ and $1 \mathrm{~ms}$ vs. $10 \mathrm{~ms}$ delay $(p=0.047)$. Similarly, it revealed a statistically significant difference between enforcing NPC vs. STLC ( $p=0.007)$, NPC vs. TOPL $(p=0.017)$, and STLC vs. TOPL $(p=0.044)$.

\section{Discussion}

To evaluate the feasibility and performance of our proposed passivity approach, we carried out an experiment in a

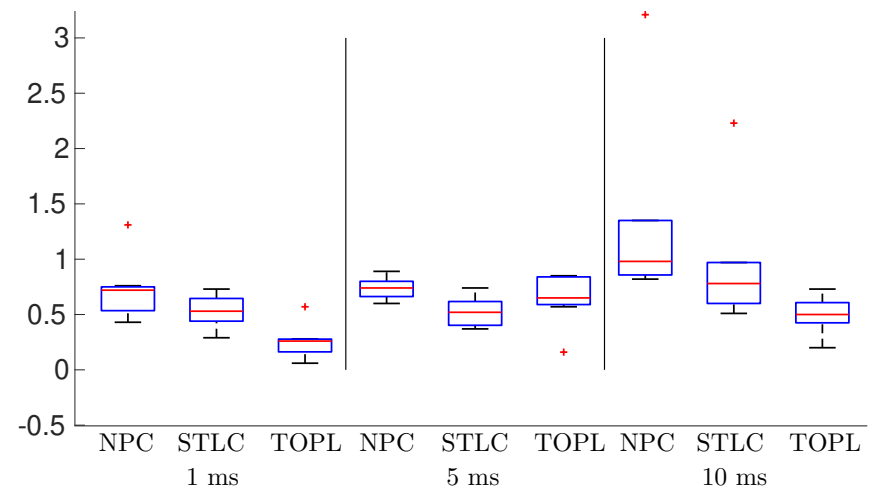

Fig. 4. Experimental evaluation. Mean accuracy error $(\mathrm{cm})$ in detecting the stiffer area within our virtual surface is plotted. simulated environment (see Sec. V-B). The user is asked to palpate a virtual surface and search for a circular area of 2 $\mathrm{cm}$ diameter which is stiffer than the rest. We tested the user's accuracy in finding such area considering (a) three different controllers (no controller, Franken et al. [18], our proposed passivity approach), and (b) three different communication delays between master and slave ( $1 \mathrm{~ms}, 5 \mathrm{~ms}$, and $10 \mathrm{~ms}$ ). Our hypotheses were that (i) as the communication delay increases, if no controller is implemented, the teleoperation system becomes unstable; conversely, the system remains always stable if one of the two considered passivity controllers are enforced. Moreover, as the communication delay increases, we also expect (ii) our TOPL approach to better preserve the transparency along prioritized directions with respect to the approach of Franken et al. [18], which does not make any difference between directions.

Both hypotheses were confirmed by our palpation experiment. When a delay of $10 \mathrm{~ms}$ was introduced in the system, the experiment was very difficult to complete, as several oscillations arose during the palpation. This unstable behaviour lead to a significantly worse accuracy with respect to the other conditions (see Fig. 4) as well as to a very high task completion time (not reported in the figures). The subject repeatedly complained about this oscillation-prone behavior, and he was often forced to increase the grasping force on the haptic interface handle to prevent it from vibrating uncontrollably. On the other hand, the interaction was always safe and stable when any of the two passivity controllers were in place. Between the two controllers, our approach outperformed the one presented by Franken et al. [18]. Of course, this result is not surprising, as our passivity controller makes use of additional information about the task, i.e., the importance of the different subspaces. This additional information is used by our TOPL controller to better distribute the energy available in the system, privileging forces rendered along the $z$ axis with respect to those rendered along the 
other axes. Fig. 3 clearly shows this difference. Whenever it is needed to reduce transparency to preserve stability, Franken et al. [18] corrects the forces along all axis in a very similar way. Conversely, our controller corrects very little along the privileged $z$ axis, while it significantly corrects the forces along the other axes. This behavior resulted in a higher transparency along $z$ at the cost of sacrificing transparency along $x$ and $y$.

\section{CONCLUSIONS AND FUTURE WORK}

In bilateral robotic telemanipulation, haptic feedback plays an important role in achieving high transparency and illusion of telepresence. Unfortunately, the stability and safety of teleoperation systems with force reflection can be significantly affected by communication latency in the loop, hard contacts, relaxed grasps, and many other timevarying destabilizing factors that dramatically reduce the effectiveness of haptics in this field. To prevent unstable behaviors, researchers have exploited passivity as one of the main tools for providing a sufficient condition for stable teleoperation. Within this context, we presented an innovative optimization-based passivity control algorithm for hapticenabled bilateral teleoperation systems with multiple degrees of freedom. Our contribution focused on implementing a novel passivity layer for the existing time-domain scheme of Franken et al. [18]. While guaranteeing passivity, we ensure optimal transparency of the interaction along subsets of the environment space, designated as the most important ones for the given task. The involved optimization problem is convex and amenable to real-time implementation. We validated the feasibility and effectiveness of the proposed approach with a palpation experiment performed on a virtual environment. Results showed that our controller outperformed the timedomain scheme of Franken et al. [18] while guaranteeing the stability and safety of the system.

In the future, we will focus on improving the performance of this proposed optimal correction approach as well as running a more extensive human subject evaluation. In this respect, we plan to study how we can automatically assign priority indexes to our subspaces given one (or more) representative runs of the considered task. Moreover, we plan to run real-world experiments using a robotic manipulator such as the 7-DoF KUKA LBR robot and a grounded haptic interface such as the 7-DoF Sigma.7 device.

\section{REFERENCES}

[1] J. E. Speich and M. Goldfarb, "Implementation of loop-shaping compensators to increase the transparency bandwidth of a scaled telemanipulation system," in Proc.IEEE International Conference on Robotics and Automation, vol. 3, pp. 2886-2893, 2002.

[2] D. Lawrence, "Stability and transparency in bilateral teleoperation," IEEE Transactions on Robotics and Automation, vol. 9, pp. 624-637, Oct. 1993.

[3] G. J. Raju, G. C. Verghese, and T. B. Sheridan, "Design issues in 2-port network models of bilateral remote manipulation," in Proc. International Conference on Robotics and Automation, pp. 1316-1321 vol.3, May 1989.

[4] T. B. Sheridan, "Space teleoperation through time delay: review and prognosis," IEEE Transactions on Robotics and Automation, vol. 9, pp. 592-606, Oct. 1993.
[5] L. Moody, C. Baber, and T. N. Arvanitis, "Objective surgical performance evaluation based on haptic feedback," Studies in Health Technology and Informatics, vol. 85, pp. 304-310, 2002.

[6] C. Pacchierotti, A. Tirmizi, G. Bianchini, and D. Prattichizzo, "Enhancing the Performance of Passive Teleoperation Systems via Cutaneous Feedback," IEEE Transactions on Haptics, vol. 8, pp. 397-409, Oct. 2015.

[7] L. Meli, C. Pacchierotti, and D. Prattichizzo, "Experimental evaluation of magnified haptic feedback for robot-assisted needle insertion and palpation," The International Journal of Medical Robotics and Computer Assisted Surgery, vol. 13, pp. n/a-n/a, Dec. 2017.

[8] C. Pacchierotti, F. Ongaro, F. v. d. Brink, C. Yoon, D. Prattichizzo, D. H. Gracias, and S. Misra, "Steering and Control of Miniaturized Untethered Soft Magnetic Grippers With Haptic Assistance," IEEE Transactions on Automation Science and Engineering, vol. 15, pp. 290306, Jan. 2018.

[9] C. R. Wagner, S. J. Lederman, and R. D. Howe, "A tactile shape display using RC servomotors," in Proc. Symposium on Haptic Interfaces for Virtual Environment and Teleoperator Systems, pp. 354-355, 2002.

[10] R. Lozano, B. Brogliato, O. Egeland, and B. Maschke, Dissipative systems analysis and control. Springer Verlag, 2000.

[11] A. v. d. Schaft, L2 - Gain and Passivity Techniques in Nonlinear Control. Communications and Control Engineering, London: Springer-Verlag, 2 ed., 2000.

[12] J.-H. Ryu, D.-S. Kwon, and B. Hannaford, "Stable teleoperation with time-domain passivity control," IEEE Transactions on Robotics and Automation, vol. 20, pp. 365-373, Apr. 2004.

[13] B. Hannaford and J.-H. Ryu, "Time-domain passivity control of haptic interfaces," IEEE Transactions on Robotics and Automation, vol. 18, pp. 1-10, Feb. 2002.

[14] G. Niemeyer and J.-J. E. Slotine, "Telemanipulation with Time Delays," The International Journal of Robotics Research, vol. 23, pp. 873-890, Sept. 2004.

[15] J.-P. Kim and J. Ryu, "Robustly Stable Haptic Interaction Control using an Energy-bounding Algorithm," The International Journal of Robotics Research, vol. 29, pp. 666-679, May 2010.

[16] J.-H. Ryu, Y. S. Kim, and B. Hannaford, "Sampled- and continuoustime passivity and stability of virtual environments," IEEE Transactions on Robotics, vol. 20, pp. 772-776, Aug. 2004.

[17] J.-H. Ryu, C. Preusche, B. Hannaford, and G. Hirzinger, "Time domain passivity control with reference energy following," IEEE Transactions on Control Systems Technology, vol. 13, pp. 737-742, Sept. 2005.

[18] M. Franken, S. Stramigioli, S. Misra, C. Secchi, and A. Macchelli, "Bilateral Telemanipulation With Time Delays: A Two-Layer Approach Combining Passivity and Transparency," IEEE Transactions on Robotics, vol. 27, pp. 741-756, Aug. 2011.

[19] F. Ferraguti, N. Preda, A. Manurung, M. Bonfe, O. Lambercy, R. Gassert, R. Muradore, P. Fiorini, and C. Secchi, "An Energy Tank-Based Interactive Control Architecture for Autonomous and Teleoperated Robotic Surgery," IEEE Transactions on Robotics, vol. 31, pp. 1073-1088, Oct. 2015.

[20] K. Salisbury, D. Brock, T. Massie, N. Swarup, and C. Zilles, "Haptic rendering: Programming touch interaction with virtual objects," in Proc. Symposium on Interactive 3D graphics, pp. 123-130, ACM, 1995.

[21] I. Desai, A. Gupta, and D. Chakraborty, "Transparency enhancement of haptic interface using model matching approach," in Proc. Indian Control Conference, pp. 399-404, Jan. 2016.

[22] M. C. J. Franken, S. Stramigioli, R. Reilink, C. Secchi, and A. Macchelli, "Bridging the gap between passivity and transparency," in Proc. Robotics: Science and Systems, July 2009.

[23] N. Hogan, "Controlling impedance at the man/machine interface," in Proc. International Conference on Robotics and Automation, pp. 16261631 vol.3, May 1989. 